\title{
ON NORM-ATTAINABLE FUNCTIONALS IN BANACH SPACES
}

\author{
BENARD OKELO \\ Institute of Mathematics, University of Muenster, Einsteinstr. 62, 48149-Muenster, Germany \\ Corresponding author: bnyaare@yah00.com \\ Received Jun. 7, 2020
}

\begin{abstract}
АвsтRACт. In this paper, we give conditions for norm-attainability of linear functionals in Banach spaces. We show that if $\Omega \in A M$ is a bounded $\sigma$-complete abstract space and $B(\Omega)$ is an orderclosed Banach space, then $\varphi \in \Omega^{*}$ is norm-attainable if and only if there exists a subspace $E$ of $\Omega$ such that $\varphi^{+}=\left.\varphi\right|_{E}$ and $\varphi^{-}=-\left.\varphi\right|_{E^{\perp}}$. Moreover, we prove that $\varphi \in \operatorname{ext} B\left(\Omega^{*}\right)$ if and only if $\varphi(x) \varphi(y)=0$, for all $x, y \in \Omega$ satisfying $x \wedge y=0$.

2010 Mathematics Subject Classification. 47B47; 47A30.
\end{abstract}

Key words and phrases. norm-attainability; functional; Banach space; abstract space; Banach lattice.

\section{InTROduction}

Norm-attainability is also a property which has been give keen attention in Banach spaces [7] and [9]. This property still remains very important as it has a lot of open questions which are unanswered particulary when a super class of Hilbert space operators called supraposinormal operators [6] are considered. In [1] the authors characterized the norm property for elementary operators and gave conditions under which a general elementary operator is norm-attainable. In-depth characterization of norm-attainable operators has also been done in details with considerations given to other properties like orthogonality (see [4] - [8] and the references therein). Regarding derivations, authors in [8] showed that if $V_{\Gamma}$ and $W_{\Gamma}$ are $\Gamma$-Banach algebras and $\delta$ an $\alpha$-inner derivation, then $\delta$ is norm-attainable if and only if the adjoint, $\delta^{*}$, of $\delta$ is normattainable. Moreover, as a consequence they proved that if $\delta_{N}^{1}$ and $\delta_{N}^{2}$ are norm-attainable then $\delta_{N}$ is norm attainable if either $\delta_{N}^{1}$ and $\delta_{N}^{2}$ or both are zero derivations and $\delta_{N}^{1}$, and $\delta_{N}^{2}$ 
are $\alpha$-inner derivation and $\alpha^{\prime}$-inner derivation respectively. In this paper, we characterize the notion of norm-attainability for linear functionals. We give necessary and sufficient conditions for norm-attainability of linear functionals in Banach spaces.

\section{Preliminaries}

In this section, we outline preliminary concepts which are useful in the sequel.

Definition 2.1. ( [5], Definition 1.1) An operator $A \in B(H)$ is said to be norm-attainable if there exists a unit vector $x_{0} \in H$ such that $\left\|A x_{0}\right\|=\|A\|$. The set of all norm-attainable operators on a Hilbert space $H$ is denoted by $N A(H)$.

Definition 2.2. ( [2]) Let $\Omega$ be a Banach lattice then $\Omega$ is an abstract $M$ space i.e. $\Omega \in A M$ if $x \wedge y=0$ implies $\|x+y\|=\max \{\|x\|,\|y\|\}$. Also $\Omega$ is abstract L space i.e. $\Omega \in A L$ if $x \vee y=0$ implies $\|x+y\|=\|x\|+\|y\|$.

Definition 2.3. ( [3])Let $\Omega$ be a Banach lattice. Then $\Omega \in A M$ implies $\Omega^{*} \in A L$ and $\Omega \in A L$ implies $\Omega^{*} \in A M . \Omega \in A M$ if and only if for any $x, y \in \Omega, x, y \geq 0$ implies $\|x \vee y\|=\max \{\|x\|,\|y\|\}$. $\Omega \in A L$ if and only if for any $x, y \in \Omega, x, y \geq 0$ implies $\|x+y\|=\|x\|+\|y\|$.

Definition 2.4. ( [2]) Let $\Omega$ be a Banach lattice. Then $\Omega$ is said to be $\sigma$ complete, if for every order bounded sequence $\left\{x_{n}\right\} \in \Omega, \vee_{n \geq 1} x_{n}$ exists in $\Omega$. Also $\Omega$ is said to be bounded $\sigma$ complete, provided that the any norm bounded and order monotone sequence in $\Omega$ is order convergent.

Definition 2.5. ( [2])Let $\Omega$ be a Banach space. An element $x \in D(\Omega)$ is called an extreme point of $B(\Omega)$ if $x=\lambda y+(1-\lambda) z, y, z \in B(\Omega)$ and $\lambda \in(0,1)$, imply $y=z$. In this case, we write $x \in \operatorname{ext} B(\Omega)$.

\section{NORM-ATTAINABILITY FOR FUNCTIONALS}

In this section, we characterize norm-attainability of functionals in Banach spaces. We regard $H^{*}$ the dual space of a Hilbert space $H$ to be non-zero throughout this section unless otherwise stated. Let $\varphi \in H^{*}$. Then $\varphi$ is said to be norm-attainable at $\frac{\varphi}{\|\varphi\|}$ if there exists $T \in B(H)$ such that $\langle\varphi, T\rangle=\|\varphi\| .\|T\|>0 . \frac{\varphi^{*}}{\left\|\varphi^{*}\right\|}$ is called a support for $\varphi$. The following proposition shows that any functional is norm-attainable in non-zero dual spaces.

Proposition 3.1. Let $B(W)$ be the set of all bounded linear maps on an Orlicz space $W$ then every $\varphi \in H_{+}^{*}$ is norm-attainable on $B(W)$. 
Proof. Let $W$ be a bounded linear Orlicz space and $b_{n} \in W$ such that $b_{n}$ is monotone decreasing to 0 . Given $J_{n} \in B(W)$ we have $\varphi\left(J_{n}\right)>\|\varphi\|-b_{n}$ since $\pi\left(J_{n}\right) \leq 1<\infty$ and $\varphi\left(W_{0}\right)=\{0\}$. Suppose that $\pi\left(J_{n}\right) \leq 2^{-n}$ and let $J(t)=\sup \left|J_{n}(t)\right|$. Then $\pi(J) \leq \sum_{n=1}^{\infty} \pi\left(J_{n}\right) \leq 1$, that is, $J \in B(W)$ and $\varphi(J) \geq \sup _{n}\left(\left|J_{n}\right|\right)=\|\varphi\|$, because $\varphi \in H_{+}^{*}$.

Theorem 3.1. Let $B(W)$ be the set of all bounded linear maps on an Orlicz space $W$ then $\varphi \in H^{*}$ is norm-attainable on $B(W)$ if and only there exists $L$ in a subspace $\mathcal{C}$ of $B(W)$ such that $\varphi^{+}=\left.\varphi\right|_{L}$ and $\varphi^{-}=\left.\varphi\right|_{G \backslash L}$.

Proof. It is known that we have $P, Q \in B(W)$ such that $\left.\varphi\right|_{L}(P) \varphi^{*}\left\|\varphi^{+}\right\|$and $\left.\varphi\right|_{G \backslash L}(Q)=\left\|\varphi^{-}\right\|$ by Proposition 3.1. Assume that $\pi\left(\left.P\right|_{L}\right) \leq \frac{1}{2}$ and $\pi\left(\left.Q\right|_{G \backslash L}\right) \leq \frac{1}{2}$. Then $\pi\left(\left.P\right|_{L}-\left.Q\right|_{G \backslash L}\right) \leq$ $\pi(P)+\pi(Q) \leq 1$. Indeed, $\left.P\right|_{L}-\left.Q\right|_{G \backslash L} \in L(W)$ and $\varphi\left(\left.P\right|_{L}-\left.y\right|_{G \backslash L}\right)=\left\|\varphi^{+}\right\|+\left\|\varphi^{-}\right\|=\|\varphi\|$. Suppose that $P \in B(W)$ satisfies $\varphi(P)=\|\varphi\|$. Let $L=\{\in G: P(l) \geq 0\}$ then we show that $\left.\varphi\right|_{L},-\left.\varphi\right|_{G \backslash L} \in H_{+}^{*}$. Now, if $\left.\varphi\right|_{L} \in H_{+}^{*}$, then there exists $Q \in W^{+}$. If $\left.\varphi\right|_{L \notin H^{\prime}}$ then there exists $Q \in W^{+}$such that $\left.\varphi\right|_{L}(Q)<0$. Now $\varphi$ being singular, assume $\pi(Q) \leq \frac{1}{2}$ and $\pi(P) \leq \frac{1}{2}$. Then $J=\left.P\right|_{G \backslash L}-\left.Q\right|_{L} \in B(W)$ and so, $\left\|\varphi^{-}\right\| \geq \varphi^{-}(-J)=-\varphi^{+}(J)+\varphi(J) \geq \varphi(J)=$ $\varphi\left(\left.P\right|_{G \backslash L}-\left.\varphi\right|_{L}(Q)\right)>\varphi\left(\left.P\right|_{G \backslash L}\right)$. This is contrary to our earlier assumption. Lastly, $\|\varphi\|=$ $\left\|\varphi^{+}\right\|+\left\|\varphi^{-}\right\|>\varphi\left|\left(\left.P\right|_{L}\right)+\varphi\left(\left.P\right|_{G \backslash L}\right)=\varphi\right|(P)=\|\varphi\| .\left(\left.\varphi\right|_{G \backslash L}\right) \in H_{1}^{*}$ follows analogously.

Corollary 3.1. Let $\varphi \in H^{*}$ is norm-attainable at $P \in B(W)$, then $\varphi\left(\left.P\right|_{A}\right)\left\|\left.\varphi\right|_{A}\right\|$ for all $A \in \mathcal{C}$.

Proof. $\|\varphi\|=\left\|\left.\varphi\right|_{A}\right\|+\left\|\left.\varphi\right|_{G \backslash A}\right\| \geq\left.\varphi\right|_{A}(P)+\left.\varphi\right|_{G \backslash A}(P)=\varphi(P)=\|\varphi\|$ is enough.

Theorem 3.2. Let $\varphi \in H^{*}$ be singular then the set of all such $\varphi$ is dense in $H^{*}$.

Proof. Let $\varphi \in H^{*}$ be singular and $\epsilon>0$ be given. Then we have $L \in \mathcal{C}$ such that $\left\|\left.\varphi^{+}\right|_{G \backslash L}\right\|<\epsilon$ and $\left\|\left.\varphi^{-}\right|_{L}\right\| \leq \epsilon$. Suppose that $\psi=\left.\varphi^{+}\right|_{L}-\left.\varphi^{-}\right|_{G \backslash L}$. Then by Theorem $3.1 \psi$ is norm-attainable. Also, $\|\varphi-\psi\| \leq\left\|\varphi^{+}-\left.\psi\right|_{l}\right\|+\left\|\varphi-\left.\psi\right|_{G \backslash l}\right\|=\left\|\left.\varphi^{+}\right|_{G \backslash l}\right\|+\left\|\left.\varphi^{-}\right|_{l}\right\|<2 \epsilon$.

Theorem 3.3. Let $B(W)$ be the set of all bounded linear maps on an Orlicz space $W$. Then $\phi=$ $\chi+\varphi\left(0 \neq \chi \in H_{0}^{*}, \varphi \in H^{*}\right)$ is norm-attainable at $P \in B(W)$ if and only if $\pi(P)=1, \varphi(P)=\|\varphi\|$ and $\int_{G} k \chi(t) P(t) d t=\pi(x)+\omega(k \chi)$, where $k \in K_{N}(\chi)=\left\{k: k^{-1}[1+\omega(k \chi)]=\|\chi\|_{N}^{0}\right\}$.

Proof. From the statement of the theorem we have $\|\phi\|^{0}=f(P)=k^{-1}\langle k \chi, \quad \chi\rangle+\varphi(P) \leq$ $k^{-1}[\pi(P)+\omega(k \chi)]+\varphi(P) \leq k^{-1}[1+\omega(k \chi)]+\|\varphi\|=\|\chi\|_{N}^{0}+\|\phi\|=\|\phi\|^{0}$. The converse follows from the fact that $\phi$ is singular from Theorem 3.2 and an assertion from Proposition 3.1. 
At this point, we consider norm-attainable functionals in Banach lattices. We denote an abstract $L$ space and abstract $M$ space by $A L$ and $A M$ respectively. For details on $A L$ and $A M$ see [2]. We state the following lemma.

Lemma 3.1. Let $\Omega \in A L$ and $\varphi \in B\left(\Omega^{*}\right)$. Then the following are equivalent.

(i). $\varphi$ is norm-attainable.

(ii). Both $\varphi^{+}$and $\varphi^{-}$are norm-attainable.

(iii). $\varphi^{+}$or $\varphi^{-}$is norm one.

Proof. $(i) \Rightarrow(i i)$. Choose $x \in B(\Omega)$ such that $\varphi(x)=\|\varphi\|=1$. From

$$
\begin{aligned}
1=\|\varphi\| & =\varphi(x)=\varphi^{+}\left(x^{+}\right)+\varphi^{-}\left(x^{-}\right)-\varphi^{+}\left(x^{-}\right)-\varphi^{-}\left(x^{+}\right) \\
& =\left\|\varphi^{+}\right\|\left\|x^{+}\right\|+\left\|\varphi^{-}\right\|\left\|x^{-}\right\|-\varphi^{+}\left(x^{-}\right)-\varphi^{-}\left(x^{+}\right) \\
& =\|\varphi\|\left(\left\|x^{+}\right\|+\left\|x^{-}\right\|\right)=\|\varphi\|\|x\|=\|\varphi\|=1
\end{aligned}
$$

we obtain $\varphi^{+}\left(x^{+}\right)=\left\|\varphi^{+}\right\|\left\|x^{+}\right\| ; \quad \varphi^{-}\left(x^{-}\right)=\left\|\varphi^{-}\right\|\left\|x^{-}\right\|$and $\varphi^{+}\left(x^{-}\right)=\varphi^{-}\left(x^{+}\right)=0$ since $\varphi^{ \pm}\left(x^{ \pm}\right) \leq\left\|\varphi^{ \pm}\right\|\left\|x^{ \pm}\right\|$and $\varphi^{ \pm},\left(x^{ \pm}\right)$are non-negative.

$(i i) \Rightarrow($ iii $)$. This follows obviously.

$($ iii $) \Rightarrow(i)$. We suppose that $\varphi^{+}$is norm one and norm-attainable. choose $x \in B\left(\Omega^{+}\right)$such that $\varphi^{+}(x)=\|\varphi\|=1$. We have $\varphi^{-}(x)=0$. Indeed, $1=\|\varphi\| \geq|\varphi|(|x|) \geq \varphi^{+}(x)+\varphi^{-}(|x|)=$ $1+\varphi^{-}(|x|) \geq 1$, which implies that $\varphi^{-}\left(x^{+}\right)=\varphi^{-}\left(x^{-}\right)=0$. Hence, $\varphi(x)=\varphi^{+}(x)=\|\varphi\|=1$.

Theorem 3.4. Let $\Omega \in A L$ and $\theta \leq \varphi \in B\left(\Omega^{*}\right)$. Then the following are equivalent.

(i). $\varphi$ is norm-attainable.

(ii). There exists $\theta \leq x \neq \theta$ such that $\varphi(y)=\|y\|$ for all $\bar{E}_{\Omega}$, the norm closure of $E_{\Omega}$ where $E_{\Omega}=\{y \in \Omega: \theta \leq y \leq n x$, for some $n>0\}$.

(iii). There exists $\theta \neq x \in \Omega^{+}$such that among $B\left(\Omega^{*}\right)=\left\{\psi \in \Omega^{*} ;\|\psi\|=1\right\}, \varphi$ is maximal on $\bar{E}_{\Omega}$.

Proof. $(i) \Rightarrow($ ii $)$. Choose $x \in B(\Omega)$ satisfying $\varphi(x)=\|x\|=1$. We have $x \in \theta$ for $1=\varphi(x)=$ $\varphi\left(x^{+}\right)=\varphi\left(x^{+}\right)-\varphi\left(^{-}\right) \leq \varphi\left(^{+}\right) \leq\left\|x^{+}\right\|-\left\|x^{-}\right\| \leq 1$ by Lemma 3.1 which implies $\left\|x^{-}\right\|=0$. Now if we consider $y \in \bar{E}_{\Omega}$, we need to prove that $\varphi(y)=\|y\|$. Since $\varphi$ is continuous, let $y \in \bar{E}_{\Omega}$, i.e $\theta \leq y \leq n x$ for some $n \geq 0$. But since $n=\varphi(n x)=\varphi(n x-y)+\varphi(y) \leq\|n x-y\|+\|y\|=$ $\|n x\|=n$, we have $\varphi(y)=\|y\|$.

$(i i) \Rightarrow(i i i)$. Follows trivially from $(i i) \Rightarrow(i i i)$ in Lemma 3.1 and abstractness of $A L$. 
$($ iii $) \Rightarrow(i)$. Let $\varphi$ be maximal in $B\left(\Omega^{*}\right)$ on $\bar{E}_{\Omega}$ for some $\theta \neq x \in \Omega^{+}$. Choose $\psi \in B\left(\Omega_{*}\right)$ such that $\psi(x)=\|x\|$, then by $\varphi(x) \geq \psi(x)=\|x\|$, it is clear that $\varphi$ is norm-attainable at $x /\|x\|$.

Proposition 3.2. Let $\Omega \in A M$ be $\sigma$-complete and $\varphi \in \Omega^{*}$. Then for any $\epsilon>0$, there exists a subspace $E$ of $\Omega=E+E^{\perp}$ and $\left\|\left.\varphi^{+}\right|_{E^{\perp}}\right\|<\epsilon,\left\|\left.\varphi^{-}\right|_{E}\right\|<\epsilon$.

Proof. Choose $x \in D(\Omega)$ with the property that $\varphi^{+}(x)>\|\varphi\|-\epsilon$, and $E=\left(x^{\perp}\right)^{\perp}$. Then $x^{+} \in E, x^{-} \in E^{\perp}$ and $\Omega=E+E^{\perp}$. Furthermore, for $x \in D(\Omega)$ we have that $\left\|\left.\varphi^{+}\right|_{E}\right\|+\left\|\left.\varphi^{+}\right|_{E^{\perp}}\right\|+$ $\left\|\left.\varphi^{-}\right|_{E}\right\|+\left\|\left.\varphi^{-}\right|_{E^{\perp}}\right\|=\left\|\varphi^{+}\left|\|+\| \varphi^{+}\|=\| \varphi \|<\varphi(x)+\epsilon=\varphi^{+}\right|_{E}(x)+\left.\varphi^{+}\right|_{E^{\perp}}(x)-\left.\varphi^{-}\right|_{E}(x)-\right.$ $\left.\varphi^{+}\right|_{E^{\perp}}(x)+\epsilon$ because $\left.\varphi^{+}\right|_{E^{\perp}}(x) \leq 0$ and $\left.\varphi^{-}\right|_{E}(x) \geq 0$. So, we conclude that $\left\|\left.\varphi^{+}\right|_{E^{\perp}}\right\|+\left\|\left.\varphi^{-}\right|_{E}\right\|=$ $\left\|\varphi^{+}\left|\|-\| \varphi^{+}\right|_{E}\right\|+\left\|\varphi^{-}\left|\|-\| \varphi^{-}\right|_{E}\right\| \leq\left\|\varphi^{+}\right\|-\left.\varphi^{+}\right|_{E}(x)+\left\|\varphi^{-}\right\|-\left.\varphi^{-}\right|_{E^{\perp}}(x)<\left.\varphi^{+}\right|_{E^{\perp}}(x)-\left.\varphi^{-}\right|_{E}(x)+$ $\epsilon \leq \epsilon$. This completes the proof as required.

Theorem 3.5. Let a Banach lattice $\Omega$ be bounded $\sigma$-complete and $B(\Omega)$ order-closed, then every positive bounded linear $\varphi \in \Omega^{*}$ is norm-attainable.

Proof. Consider $x_{n}(\geq 0) \in D(\Omega)$ such that $\varphi\left(x_{n}\right) \rightarrow\|\varphi\|$. Since $\Omega$ is bounded $\sigma$-complete and $B(\Omega)$ is closed under order, $y=\vee_{n}\left(x_{n}\right)$ exists in $\Omega$ and $\|y\|=1$. Hence, $y \geq x_{n} \geq 0$ and $\varphi \geq 0$ implies $\|\varphi\| \geq \varphi(y) \geq \varphi\left(x_{n}\right) \rightarrow\|\varphi\|$. So, $x \in D(\Omega)$ exists which satisfies the norm-attainability condition, $\varphi(x)=\|\varphi\|$, for functionals and this completes the proof.

Theorem 3.6. Let $\Omega \in A M$ be bounded $\sigma$-complete and $B(\Omega)$ order-closed, then $\varphi \in \Omega^{*}$ is normattainable if and only if there exists a subspace $E$ of $\Omega$ such that $\varphi^{+}=\left.\varphi\right|_{E}, \varphi^{-}=-\left.\varphi\right|_{E^{\perp}}$.

Proof. Necessity. Let $x \in B(\Omega)$ be having the property that $\varphi \mid(x)=\|\varphi\|$, and define $E=\left(x^{-}\right)^{\perp}$. Then $\Omega=E+E^{\perp}$ and $x^{+} \in E, x^{-} \in E^{\perp}$. Now, $\|\varphi\|=\left\|\left.\varphi\right|_{E}\right\|+\left\|\left.\varphi\right|_{E^{\perp}}\right\|$; we need to show that $\varphi^{+}=\left.\varphi\right|_{E}$ and $\varphi^{-}=-\left.\varphi\right|_{E^{\perp}}$, it is enough that $\left.\varphi\right|_{E} \geq 0$ and $-\left.\varphi\right|_{E^{\perp}} \geq 0$. Indeed, if $\varphi_{E}(y)<0$ for some $y(\geq 0) \in D(\Omega)$ then we let $y \in E$. So, $z=-x^{-}-y$ satisfies $\|z\|=\max \{\|x\|,\|y\|\}=1$ and hence, $\left\|\varphi^{-}\right\|=\varphi^{-}(-z)=\varphi(z)-\varphi^{+}(z) \geq \varphi(z)=\left.\varphi\right|_{E^{\perp}}\left(-x^{-}\right)-\left.\varphi\right|_{E}(y)>\left.\varphi\right|_{E^{\perp}}\left(-x^{-}\right)=$ $-\left.\varphi\right|_{E^{\perp}}(-x)$. Now since $\left\|\varphi^{+}\right\| \geq \varphi\left(\left.x\right|_{E}\right)=\left.\varphi\right|_{E}(x)$, this is contrary to $\|\varphi\|=\left\|\varphi^{+}\right\|+\left\|\varphi^{-}\right\|>$ $\left.\varphi\right|_{E}(x)-\left.\varphi\right|_{E^{\perp}}(x)=\varphi(x)=\|\varphi\|$. Also $-\left.\varphi\right|_{E^{\perp}} \geq 0$ follows analogously.

Sufficiency. We know that there exists $x, y(\geq 0) \in D(\Omega)$ such that $\varphi^{+}(x)=\left\|\varphi^{+}\right\|$and $\varphi^{-}(x)=$

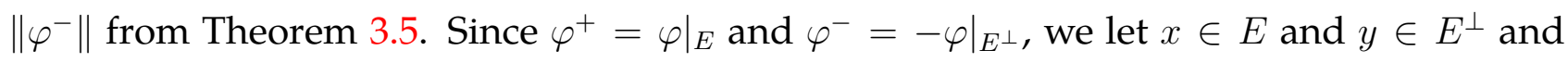
$u \in x-y$. Then $u=\|x-y\|=\max \{\|x\|,\|y\|\}=1$ and so we have $\|\varphi\|=\left\|\varphi^{+}\right\|+\left\|\varphi^{-}\right\|=$ $\varphi^{+}(x)+\varphi^{-}(y)=\left.\varphi\right|_{E}(x)+\left.\varphi\right|_{E^{\perp}}(-y)=\varphi(u)$. 
Corollary 3.2. Let $\Omega \in A M$ be a $\sigma$-complete and $\varphi \in D\left(\Omega^{*}\right)$. Then $\varphi \in \operatorname{ext} B\left(\Omega^{*}\right)$ if and only if $\varphi(x) \varphi(y)=0$ for all $x, y \in \Omega$ satisfying $x \wedge y=0$.

Proof. Necessity. If there exists $x, y \in \Omega$ satisfying $x \wedge y=0$ but $\varphi(x)>0$ and $\varphi(y)>0$, then we set $E=y^{\perp}$, and $\Omega=E+E^{\perp}$. Let $\psi=\left.\varphi\right|_{E}$ and $\tau=\left.\varphi\right|_{E^{\perp}}$. Then $\|\psi\|>0,\|\psi\|>0$ since $x \in E, y \in E^{\perp}$. Therefore, $\varphi=\|\psi\| \frac{\psi}{\|\psi\|}+\|\tau\| \frac{\tau}{\|\tau\|}$ and $\|\psi\|+\|\tau\|=\|\varphi\|=1$. Hence, $\varphi \in \operatorname{ext} B(\Omega)$.

Sufficiency. First we show $\left\|\varphi^{+}\right\|\left\|\varphi^{-}\right\|=0$. In fact, for any $\epsilon>0$, by Theorem 3.5, there exists two orthogonal subspaces $E, F \in \Omega$ such that $\Omega=E+F$ and $\left\|\left.\varphi^{-}\right|_{E}\right\|<\epsilon,\left\|\left.\varphi^{+}\right|_{F}\right\|<\epsilon$. Choose $x \in B(\Omega)$ such that $\varphi(x)>\|\varphi\|-\epsilon$, and let $x=u+v$, where $u \in E$ and $v \in F$. Then $\varphi(u) \varphi(v)=0$ since $u \wedge v=0$. If $\varphi(v)=0$ then $\|\varphi\|-\epsilon<\varphi(x)=\left.\varphi^{+}\right|_{E}(u)-\left.\varphi^{-}\right|_{E}(u) \leq$ $\left\|\left.\varphi^{+}\right|_{E}\right\|+\left\|\left.\varphi^{-}\right|_{E}\right\|<\left\|\varphi^{+}\right\|+\epsilon$. Let $\epsilon \rightarrow 0$, we find $\left\|\varphi^{-}\right\|=\|\varphi\|-\left\|\varphi^{+}\right\|=0$. Similarly, if $\varphi(u)=0$. Then $\left\|\varphi^{+}\right\|=0$. Hence, without loss of generality, we assume $\varphi=\varphi^{+}$. Let $\psi, \tau \in D\left(\Omega^{*}\right)$ satisfy $2 \varphi=\psi+\tau$. Then $2 \varphi=\left(\psi^{+}+\tau^{+}\right)-\left(\psi^{-}+\tau^{-}\right)$and hence $\|2 \varphi\|=\left\|\psi^{+}\right\|+\left\|\tau^{+}\right\|+\left\|\psi^{-}\right\|+\left\|\tau^{-}\right\|=$ $\|\psi\|+\|\tau\|=2=\|2 \varphi\|$ Thus $\psi^{+}+\tau^{+}=2 \varphi$ and $\psi^{-}=\tau^{-}=0$. Now we show $\psi=\tau=\varphi$, that is, $\varphi \in \operatorname{ext} B\left(\Omega^{*}\right)$. This follows if we prove that $\psi(y)=\tau(y)=0$ whenever $\varphi(y)=0$ this means $\varphi=a \psi=b \tau$, but $\varphi, \psi, \tau \in D\left(\Omega^{*}\right)$ and $2 \varphi=\psi+\tau$, so $a=b=1$, this means assume $y \geq 0$; then from $\psi(y) \geq 0, \tau(y) \geq 0$ and $\psi(y+\tau(y)=2 \varphi(y)=0$. We have $\psi(y)=\tau(y)=0$. For the general case, since $\varphi(y)=0$ and by the condition given in theorem $\varphi\left(y^{+}\right) \varphi\left(y^{-}\right)=0$, we have $\varphi\left(y^{+}\right)=\varphi\left(y^{-}\right)=0$ hence the condition $\psi(y)=\tau(y)=0$ follows from the first case.

\section{Conclusion}

In this work, we have established norm- attainability of functionals in Banach spaces. We have shown that a functional $\varphi \in \Omega^{*}$ is norm-attainable if and only if there exists a subspace $E$ of $\Omega$ such that $\varphi^{+}=\left.\varphi\right|_{E}$ and $\varphi^{-}=-\left.\varphi\right|_{E^{\perp}}$.

\section{ACKNOWLEDGeMENT}

The author acknowledges financial support through TWAS-DFG research Grant No. 119840. The author is also grateful to the referees for their careful reading of the manuscript and valuable comments. The author thanks the help from the editor too.

\section{Author's CONTRIBUtion}

The author contributed wholly in writing this article and declares no conflict of interest. 


\section{REFERENCES}

[1] H. K. Du, Y. Q. Wang, G. B. Gao, Norms of Elementary Operators, Proc. Amer. Soc. 36 (2008), 1337-1348.

[2] N. Dunford, J. T. Schwartz, Linear operators, Interscience, New York (1958).

[3] E. Kreyzig, Introductory Functional Analysis with Applications, John Wiley and sons, New York (1978).

[4] N. B. Okelo, J. O. Agure, D. O. Ambogo, Norms of elementary operators and characterization of normattainable operators, Int. J. Math. Anal. 24 (2010), 1197-1204.

[5] N. B. Okelo, The norm-attainability of some elementary operators, Appl. Math. E-Notes, 13 (2013), 1-7.

[6] N. B. Okelo, $\alpha$-Supraposinormality of operators in dense norm-attainable classes, Univ. J. Math. Appl. 2 (2019), 42-43.

[7] N. B. Okelo, On orthogonality of elementary operators in norm-attainable classes, Taiwan. J. Math. 24 (2020), 119-130.

[8] N. B. Okelo, M. O. Okongo, S. A. Nyakiti, On projective tensor norm and norm-attainable $\alpha$-derivations, Int. J. Contemp. Math. Sci. 5 (2010), 1969-1975 .

[9] N. B. Okelo, J. O. Agure, P. O. Oleche, Various notions of orthogonality in normed spaces, Acta Math. Sci. 33 (2013), 1387-1397. 